\title{
Industrial Process Control Using LPV
}

\author{
Musa Abdalla ${ }^{1} \&$ Tamir Shagarin ${ }^{2}$ \\ ${ }^{1}$ School of Engineering, The University of Jordan, Amman, Jordan \\ ${ }^{2}$ College of Engineering, Tafila Technical University, Tafila, Jordan \\ Correspondence: Musa Abdalla, School of Engineering, The University of Jordan, Amman, Jordan. Tel: \\ 962-6535-5000. E-mail: admin@mechatronix.us
}

Received: June 8, 2017

Accepted: July 25, 2017

Online Published: August 12, 2017

doi:10.5539/mas.v11n9p39

URL: https://doi.org/10.5539/mas.v11n9p39

\begin{abstract}
An industrial process control application of level and temperature is considered. The nonlinear mathematical model of the system is cast as a linear parameter varying (LPV) system. A linear matrix inequality (LMI) type of controller is successfully designed using the LMI unified approach to regulating both controlled variables, namely; temperature and level. The closed loop system is then implemented through computer simulation to show the effectiveness of the controller in performing the combined level-temperature regulation. Basically, this combined level and temperature industrial control application is used to demonstrate the effectiveness of post-modern controllers; in this case LMI based controllers.
\end{abstract}

Keywords: Linear Parameter Varying System (LPV), temperature control, level control, multivariable control, LMI

\section{Introduction}

Level and temperature control have a major importance in industrial applications especially in chemical processes which are widely used in Continuous Stirred Tank Reactors (CSTR), steam generators, distillation columns, etc. Classical control analysis simplifies the analysis for the problem into two decoupled linearized differential equations, however, under certain circumstances; this classical linear control analysis is turned out to be inadequate.

Currently, the main control techniques used in level and temperature control is the conventional PID controllers based on linearized model, in which they used two separate controllers which ignore all nonlinearities and dynamic coupling between the level and temperature. Also, some researchers used the system's nonlinear model and applied nonlinear control strategies; such as Model Predictive Control (MPC) and Slide Mode Control, etc.

For a single tank model, some researchers in literature have simplified the model into two decoupled equations by taking nominal value for the volume and applied it in the energy equation (Bequette, 1998) (Seborg, 2004). While for level and temperature PID Control, others have used simple proportional controllers to control the tank level and the temperature by manipulating the flow of the liquid leaving the tank and the cooling water (Coughanowr \& et. al., 2008)(Perez \& Albertos, 2004). Recent studies have used PI controller in CSTR to control the level of the tank in order to keep the volume constant, and they controlled the temperature of the outflow by using two controllers; the first PI controller placed on the reactor outflow to control the level, and the second PI controller on the cooling water flow to control the reactor temperature (Perez \& Albertos, 2004). For more demanding applications (Johansson, 1997), where three variables needed to be regulated, researchers have used PI controllers to control the level, pressure, and temperature of the juice in industrial deaeration process.

The problem in hand is basically a simultaneous control of temperature and level in a single tank, which is depicted in figure 1. Essentially the controller needs to provide two control laws; one for controlling the inlet valve (inflow) and the second one to control the heater. It is our objective to maintain a constant temperature and level despite the unknown inflow temperature and the unknown outflow rate. Hence, the controlled variables are the level of the liquid in the tank and the temperature of the liquid inside the tank, and the manipulated variables are the inlet inflow rate and the heat rate supplied to the tank.

In the literature, a simplified mathematical model is used that is composed of two linearized and decoupled differential equations, where a nominal value of the volume was taken constant in the derivation (Bequette, 1998) 
(Seborg, 2004).

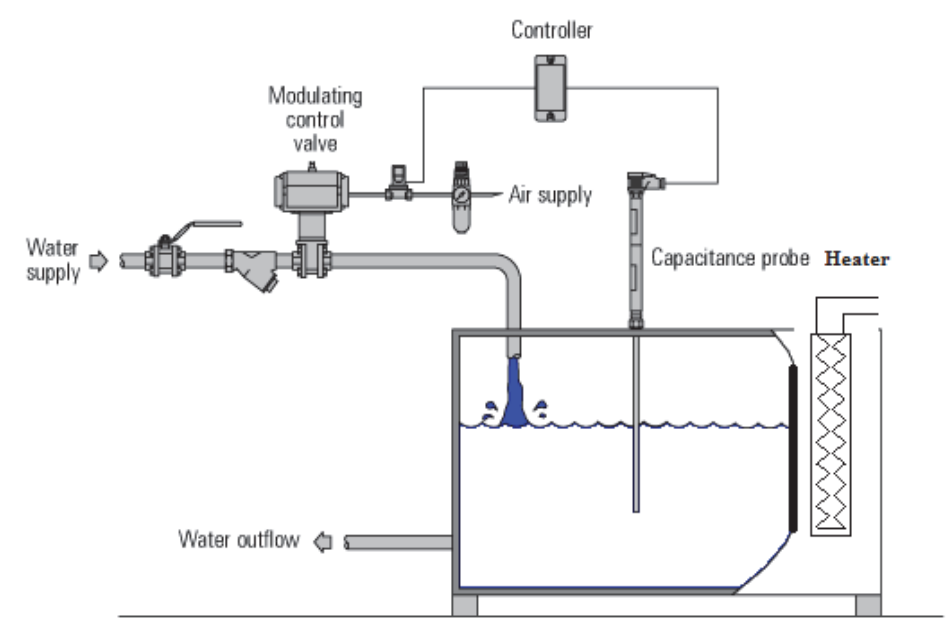

Figure 1. Level/Temperature control in a tank (Spiraxsarco, 2016)

The resulting model is basically given by

$$
\begin{aligned}
& \frac{d V}{d t}=\frac{1}{\rho}\left(w_{i}-w\right) \\
& \frac{d T}{d t}=\frac{w_{i}}{V \rho}\left(T_{i}-T\right)+\frac{Q}{V \rho C}
\end{aligned}
$$

Where $V$ is the volume of the tank, $\rho$ is the density of the liquid, $w_{i}$ is the inlet mass flow rate, $w$ is the outlet mass flow rate, $T$ is the temperature of liquid inside the tank, $T_{i}$ is the temperature of the feed stream, $Q$ is the rate of heat added to the tank and $C$ is the liquid specific heat.

An arbitrary representation of the linearized state space model around the steady state values maybe casted as $\left(T_{S}, H_{S}, F_{o s}, Q_{S}\right)$

$$
\left[\begin{array}{c}
\dot{x}_{1} \\
\dot{x}_{2}
\end{array}\right]=\left[\begin{array}{cc}
\frac{-1}{A R} & 0 \\
0 & \frac{-F_{O S}}{A H_{S}}
\end{array}\right]\left[\begin{array}{c}
x_{1} \\
x_{2}
\end{array}\right]+\left[\begin{array}{cc}
\frac{1}{A} & 0 \\
\frac{T_{O}-T_{S}}{A H_{S}} & \frac{1}{H_{S} \rho C_{p} A}
\end{array}\right]\left[\begin{array}{c}
u_{1} \\
u_{2}
\end{array}\right]
$$

Where the state variables and inputs are defined as follows

$$
x_{1}=H-H_{S}, \quad x_{2}=T-T_{S}, \quad u_{1}=F_{o}-F_{O S}, \quad u_{2}=Q-Q_{S} .
$$

There are some concerns about the aforementioned model, such as: the constant volume assumption that was used to decouple the two coupled ODEs, which will lead to a less effective controller design, the model can be used only if the level is constant and cannot be applied to applications with variable level ("Variable Holdup") depending on the demands. Also, for constant level applications, it will not estimate the temperature effectively when the system has disturbances on the level part.

A lot of studies have used conventional control techniques (PID) to control the level and temperature, but in this study, post-modern control techniques are applied to the system.

Consider the temperature and level control problem that is depicted in figure 1 and by applying energy balance on the system, the following model will be obtained, assuming that the kinetic and potential energy differences are negligible, with no shaft work is taken for the stirrer. The temperature distribution can be easily shown by the following coupled, first-order nonlinear differential equation: 


$$
\dot{T}=\frac{F_{O} T_{O}}{A H}-\frac{T}{A R}+\frac{Q}{H \rho A C_{p}}-\frac{\dot{H} T}{H}
$$

Consequently, the liquid level dynamics $H(t)$ can be captured by the following, first order, linear differential equation:

$$
\dot{H}=\frac{F_{O}}{A}-\frac{H}{A R}
$$

Substitute equation (4) into equation (3) one gets

$$
\dot{T}=\frac{F_{O} T_{O}}{A H}-\frac{F_{o} T}{A H}+\frac{Q}{H \rho A C_{p}}
$$

After some manipulations, rearranging the equations, and considering the error signals with the reference (set points $T_{\text {set }}$ and $H_{\text {set }}$ ) as the state variables, the state space representation for the problem will be

$$
\left[\begin{array}{cc}
1 & 0 \\
0 & H
\end{array}\right]\left[\begin{array}{c}
\dot{h} \\
\dot{\bar{T}}
\end{array}\right]=\left[\begin{array}{cc}
\frac{-1}{A R} & 0 \\
0 & \frac{-F_{o}}{A}
\end{array}\right]\left[\begin{array}{c}
h \\
\bar{T}
\end{array}\right]+\left[\begin{array}{cc}
\frac{1}{A} & 0 \\
\frac{T_{o}}{A} & \frac{1}{\rho A C_{p}}
\end{array}\right]\left[\begin{array}{c}
F_{o} \\
Q
\end{array}\right]+\left[\begin{array}{cc}
\frac{-1}{A R} & 0 \\
0 & \frac{-F_{o}}{A}
\end{array}\right]\left[\begin{array}{c}
H_{\text {set }} \\
T_{\text {set }}
\end{array}\right]
$$

Where: $h=H-H_{\text {set }}, \quad \bar{T}=T-T_{\text {set }}$

The extended LTI system presented in equation (6) may be written in the form

$$
\begin{aligned}
& E_{p^{\dot{x}} p}=A_{p^{x} p}+B_{p} u+D_{p^{w}} \\
& y_{p}=C_{p^{x} p}+B_{y} u+D_{y} w \\
& z_{p}=M_{p^{x}} p+\mathrm{D}_{\mathrm{z}} w
\end{aligned}
$$

Where $Z_{p}$ represents the measurements equation.

\section{Linear Parameter Varying System}

In recent years, Linear Parameter Varying (LPV) controller design has attracted many researchers of the control community (Gilbert \& et. al., 2010) (Bianchi \& et. al. 2004). LPV controller design is used to control dynamical systems with parameter variations. Typically, LPV systems are linear time-varying plants whose system matrices are functions of a varying vector of parameters.

Physical models of a system often lead to a state-space description of its dynamical behavior. The resulting state-space equations typically involve physical parameters whose values are only approximately known, as well as approximations of complex and possibly nonlinear phenomena. Consequently, the system is described by an uncertain state-space model.

$$
E \dot{x}=A x+B u \quad y=C x+D u
$$

Where the state-space matrices A, B, C, D, and E depend on uncertain and/or time-varying parameters or vary in some bounded sets of the space of matrices.

Consequently, the LPV techniques provide a systematic unified design procedure for gain-scheduled multivariable controllers. In the LPV design methodology performance, robustness and bandwidth limitations maybe are incorporated into a unified framework (Skelton \& et. al., 1997)(El Ghaoui \& Niculescu, 2000).

An LPV system has either polytopic model, that is a linear time-varying system or an affine parameter-dependent model. The process tank problem in hand has an affine parameter- dependent plant and its state space representation is given as 


$$
\begin{aligned}
& E_{p}(p) \dot{x}_{p}=A_{p}(p) x_{p}+B_{p}(p) u+D_{p}(p) w \\
& y_{p}=C_{p}(p) x_{p}+B_{y}(p) u+D_{y}(p) w \\
& z_{p}=M_{p}(p) x_{p}+\mathrm{D}_{\mathrm{z}}(p) w
\end{aligned}
$$

Consider incorporating a controller of the form

$$
\begin{aligned}
& \dot{x}_{c}=A_{c}(p) x_{c}+B_{c}(p) z \\
& u=C_{c}(p) x_{c}+D_{c}(p) z
\end{aligned}
$$

Hence, the closed loop system becomes:

$$
\begin{aligned}
& {\left[\begin{array}{cc}
E_{p} & 0 \\
0 & I_{n c}
\end{array}\right]\left[\begin{array}{l}
\dot{x}_{p} \\
\dot{x}_{c}
\end{array}\right]=\left[\begin{array}{cc}
A_{p}+E_{p} D_{c} M_{p} & B_{p} C_{c} \\
B_{c} M_{p} & A_{c}
\end{array}\right]\left[\begin{array}{c}
x_{p} \\
x_{c}
\end{array}\right]+\left[\begin{array}{c}
D_{p}+B_{p} D_{c} D_{z} \\
B_{c} D_{z}
\end{array}\right] w} \\
& y=\left[C_{p}+B_{y} D_{c} M_{p} \quad B_{y} C_{c}\right]\left[\begin{array}{l}
x_{p} \\
x_{c}
\end{array}\right]+\left[D_{y}+B_{y} D_{c} D_{z}\right] w
\end{aligned}
$$

Or in short

$$
\begin{aligned}
& E_{c l} \dot{x}=A_{c l} x+B_{c l} w \quad A_{c l}=A+B G M \quad x_{p} \in R^{n p}, x_{c} \in R^{n c}, y_{p} \in R^{n y}, \\
& y=C_{c l}+D_{c l} w \\
& \text { Where, } \quad B_{c l}=D+B G E \text { and } z \in R^{n x}, \quad w \in R^{n w}, u \in R^{n u} \\
& C_{c l}=C+H G M \quad x \in R^{n x}, \quad n_{x}=n_{p}+n_{c} \\
& D_{c l}=F+H G E
\end{aligned}
$$

Using the Real Bounded Lema from Linear Matrix Inequalities (LMI) unified approach, one may prove the following theorem (proof was omitted to save some space).

\section{Theorem I}

Consider an LPV polytopic plant; there exist an LPV controller guaranteeing quadratic $H_{\infty}$ performance $\gamma$ along all parameter trajectories in the polytope

$$
p \in \Theta=\left\{\sum_{i=1}^{r} \alpha_{i} \theta_{i}: \alpha_{i} \geq 0 ; \sum_{i=1}^{r} \alpha_{i}=1\right\}
$$

That is there exists a controller $G$ to solve the $\gamma$ - optimal $H_{\infty}$ control problem if and only if there exist matrices $X$ and $\bar{Y}$ such that the following conditions are satisfied:

$$
\begin{gathered}
{\left[\begin{array}{c}
M_{p i}^{T} \\
D_{z i}^{T}
\end{array}\right]^{\perp}\left[\begin{array}{cc}
X E_{p i}^{-1} A_{p i}+A_{p i}^{T} E_{p i}^{-T} X+C_{p i}^{T} C_{p i} & X E_{p i}^{-1} D_{p i}+C_{p i}^{T} D_{y i} \\
D_{p i}^{T} E_{p i}^{-T} X+D_{y i}^{T} C_{p i} & D_{y i}^{T} D_{y i}-\gamma^{2} I
\end{array}\right]\left[\begin{array}{c}
M_{p}^{T} \\
D_{z}^{T}
\end{array}\right]^{\perp T}<0} \\
{\left[\begin{array}{c}
B_{p i} \\
B_{y i}
\end{array}\right]^{\perp}\left[\begin{array}{cc}
A_{p i} \bar{Y} E_{p i}^{T}+E_{p i} \bar{Y} A_{p i}^{T}+D_{p i} D_{p i}^{T} & E_{p i} \bar{Y} C_{p i}^{T}+D_{p i} D_{y i}^{T} \\
C_{p i} \bar{Y} E_{p i}^{T}+D_{y i} D_{p i}^{T} & D_{y i} D_{y i}^{T}-\gamma^{2} I
\end{array}\right]\left[\begin{array}{c}
B_{p i} \\
B_{y i}
\end{array}\right]^{\perp T}<0} \\
{\left[\begin{array}{cc}
X & \gamma \\
\gamma & \bar{Y}
\end{array}\right]>0}
\end{gathered}
$$

Notes: - Proof is in the Appendix

- The smallest $\gamma$ for which a Lyapunov function exists is called the quadratic performance. This is an upper bound on the worst-case RMS gain of the system.

- In this work, we only considered polytopic LPV systems. In polytopic systems the system matrices depend affinely on a time varying vector of parameters $p$ which varies within a polytope $\Theta$ of vertices $\theta_{1}, \theta_{2}, \ldots . ., \theta_{r}$ satisfying the following equations: 


$$
\begin{aligned}
\Theta & =\sum_{i=1}^{r} \alpha_{i} \theta_{i} \\
\left(\begin{array}{ccc}
A_{p}(p)-j E_{p}(p) & B_{p}(p) & D_{p}(p) \\
C_{p}(p) & B_{y}(p) & D_{y}(p) \\
M_{p}(p) & 0 & D_{z}(p)
\end{array}\right) & =\sum_{i=1}^{r} \alpha_{i}\left(\begin{array}{ccc}
A_{p}\left(\theta_{i}\right)-j E_{p}\left(\theta_{i}\right) & B_{p}\left(\theta_{i}\right) & D_{p}\left(\theta_{i}\right) \\
C_{p}\left(\theta_{i}\right) & B_{y}\left(\theta_{i}\right) & D_{y}\left(\theta_{i}\right) \\
M_{p}\left(\theta_{i}\right) & 0 & D_{z}\left(\theta_{i}\right)
\end{array}\right) \\
\alpha_{i} \geq & 0 \text { and } \sum_{i=1}^{r} \alpha_{i}=1
\end{aligned}
$$

The system matrices at any instant can be spanned by the polytope of matrices whose vertices are the images of the vertices $\theta_{i}$. This convex formulation reduces the infinite number of system matrices needed for design to a finite number, and therefore only a finite set of LMI's should be considered. It is also interesting to note that at a specified 'frozen' $p$ the LPV system reduces to an LTI system with a local type of properties about some particular value of $p$, while the LPV system properties are global because they capture the behavior of the system along all possible trajectories $p$.

Finally, the parameter-dependent models are well-suited for Lyapunov-based analysis and synthesis and are easily converted to linear-fractional uncertainty models for small-gain-based design, using the following notation

$$
S(p)=\left[\begin{array}{cc}
A(p)-j E(p) & B(p) \\
C(p) & D(p)
\end{array}\right], \ldots \ldots . . S_{i}=\left[\begin{array}{cc}
A_{i}-j E_{i} & B_{i} \\
C_{i} & D_{i}
\end{array}\right]
$$

The affine dependence on $p$ is written more compactly in system matrix terms as: $S(p)=S_{0}+p_{1} S_{1}+\ldots+p_{n} S_{n}$.

The system "coefficients" $S_{0}, \ldots, S_{n}$ fully characterizes the dependence on the uncertain parameters $p_{1}, \ldots, p_{n}$. Note that $S_{0}, \ldots, S_{n}$ need not represent meaningful dynamical system's parameters. Only their combination $S(p)$ is a relevant description of the problem.

\section{Numerical Example}

Consider the linearized temperature and level single tank system with the following parameters: Cross section area of the tank $A=0.4 \mathrm{~m}^{2}$, Density of the Water $\rho=1000 \mathrm{~kg} / \mathrm{m}^{3}$, Specific heat of the water $C_{p}=4.179 \mathrm{~kJ} /$ $K g K^{o}$, Steady state value of heat power $Q_{s}=500 \mathrm{kw}$, Steady state value of water level $H_{s}=1 \mathrm{~m}$, Initial temperature of the water $T_{o}=300^{\circ} \mathrm{K}$ and Valve resistance $R=1000 \mathrm{~s} / \mathrm{m}^{2}$.

Let's consider a numeric representative example for the combined temperature-level problem to examine the closed loop response with the LPV controller. Assuming the process setup that is depicted in figure 1, then the state space representation of the tank model becomes:

Plant:

$$
y_{p}=\left[\begin{array}{llll}
0 & 0 & 0 & 0 \\
0 & 0 & 0 & 0 \\
0 & 0 & 1 & 0 \\
0 & 0 & 0 & 1
\end{array}\right]\left[\begin{array}{c}
H \\
T \\
e_{H} \\
e_{T}
\end{array}\right]+\left[\begin{array}{ll}
1 & 0 \\
0 & 1 \\
0 & 0 \\
0 & 0
\end{array}\right]\left[\begin{array}{c}
F_{o} \\
Q
\end{array}\right]+\left[\begin{array}{ll}
0 & 0 \\
0 & 0 \\
0 & 0 \\
0 & 0
\end{array}\right]\left[\begin{array}{c}
H_{S} \\
T_{S}
\end{array}\right]
$$

\section{Measurements:}

$$
z_{p}=\left[\begin{array}{llll}
1 & 0 & 0 & 0 \\
0 & 1 & 0 & 0 \\
0 & 0 & 0 & 0 \\
0 & 0 & 0 & 0
\end{array}\right]\left[\begin{array}{c}
H \\
T \\
e_{H} \\
e_{T}
\end{array}\right]+\left[\begin{array}{ll}
0 & 0 \\
0 & 0 \\
0 & 0 \\
0 & 0
\end{array}\right]\left[\begin{array}{c}
F_{o} \\
Q
\end{array}\right]+\left[\begin{array}{cc}
-1 & 0 \\
0 & -1 \\
0 & 0 \\
0 & 0
\end{array}\right]\left[\begin{array}{c}
H_{s} \\
T_{s}
\end{array}\right]
$$

The parameter variations:

$$
P_{1} \in[0.1,2] ; \quad P_{2} \in[-0.2,-3]
$$

The parameter range for $\Theta$ is the box $[0.1,2] \times[-0.2,-3]$; consequently this box has four vertices $(0.1,-0.2)$, 
$(0.1,-3),(2,-0.2)$, and $(2,-3)$

A Matlab code was generated to solve the LMI's presented in theorem (I) using the LMI toolbox and the Bisection method to minimize $\gamma$. The optimal value for the $H_{\infty}$ performance index $\gamma$ was found to be 8.4999. This result is clearly depicted in figure 2 . The corresponding feasible pair $(X, \bar{Y})$ that satisfies the LMI's in theorem (I) at the four vertices is given by

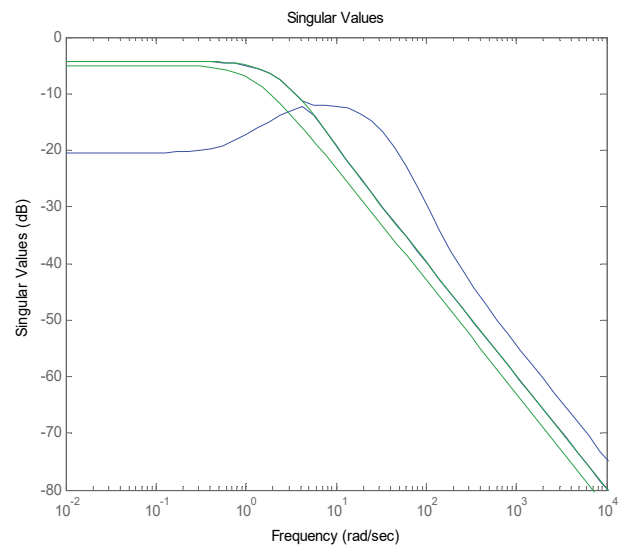

Figure 2. System's singular values

$$
X=\left[\begin{array}{cccc}
35.3916 & 0 & -10.5419 & 0 \\
0 & 55.7275 & 0 & -15.3676 \\
-10.5419 & 0 & 19.1786 & 0 \\
0 & -15.3676 & 0 & 32.1071
\end{array}\right] \quad \text { and } \quad \bar{Y}=\left[\begin{array}{cccc}
55.6873 & 0.0000 & 30.8192 & 0.0000 \\
0.0000 & 48.8968 & -0.0000 & 36.3938 \\
30.8192 & -0.0000 & 43.2532 & -0.0000 \\
0.0000 & 36.3938 & -0.0000 & 38.8944
\end{array}\right]
$$

Figure 3 shows the closed loop response for the model with the LPV controller. The top graphs show the level variation and the generated control law by the controller, while the bottom graphs show the temperature variation and the heater input.
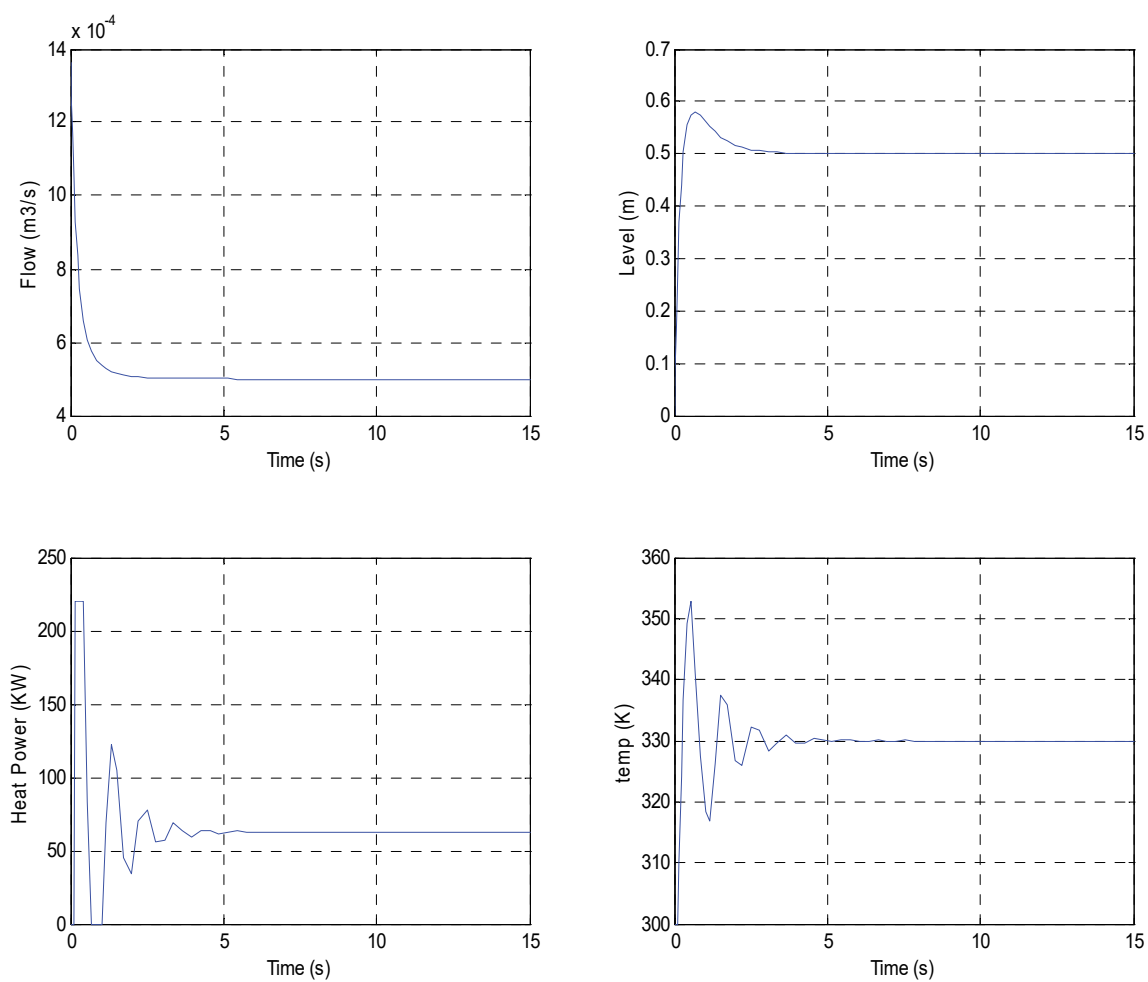

Figure 3. Tank Level/Temperature response for a small scale system 
The performance of controller seems to be reasonable good, in spite of the relatively large value for $\gamma$, as it is clearly shown that we have slight overshoot and oscillations before settling. Please note that the speed of response for the LMI based LPV controller is adequate, but this causes the system to slightly overshoot. Finally, the LMI objective function needs to be further tuned and adjusted to put more emphasis on overshoot and to relax the response speed.

\section{Conclusions}

A multivariable control problem, which combines temperature and level control, is presented. The applications for such a system are widely popular in the process industry. The system mathematical model for the combined system was fully derived verified and validated through computer simulations (Matlab/Simulink).

Linear Matrix Inequality (LMI) methods were presented to design a controller for the combined temperature and level application. The system was treated as an Linear Parameter Varying System (LPV) assuming slow changing measured parameters. Then the LMI optimal problem and constraints were successfully formulated and derived.

The LMI controller was incorporated with the system and it showed good results. Performance could be further enhanced by reducing the relatively large value of the performance index, which is mainly due to the violation of the slow varying constraint assumption.

\section{References}

Abdalla, M., et al. (2001). Fault Detection and Isolation Filter Design for Linear Parameter Varying Systems, Proceedings of the American Control Conference, Arlington, USA.

Abdullah, A., \& Zribi, M. (2016). Control Schemes for a Quadruple Tank Process, Int. J. Computing and Communication, 7(4), 594-605.

Bequette, B. W. (1998). Process Dynamics: Modeling, Analysis, and Simulation ( $1^{\text {st }}$ Ed.). New Jersey: Prentice Hall.

Bianchi, W., et al. (2004). Control of variable-speed wind turbines by LPV gain scheduling. Wind Energy, $7(2004), 1-8$.

Coughanowr, D., et al. (2008). Process Systems Analysis and Control ( $3^{\text {rd }}$ Ed.). New York: McGraw-Hill.

El Ghaoui, L., \& Niculescu, S. (2000). Advances in Linear Matrix Inequality Methods in Control ( ${ }^{\text {st }}$ Edition). Philadelphia: Advances in Design and Control, SIAM.

Gahinet, P., et al. (1995). Uncertain Systems ( $1^{\text {st }}$ ed.). USA: LMI Control Toolbox Manual, Mathworks Inc.

Ge, S., Sun, Z., \& Lee, T. (2001). Nonregular feedback linearization for a class of second-order nonlinear systems, Automatica, 37, 1819-1824.

Gilbert, H., et al. (2010). Polynomial LPV Synthesis Applied to Turbofan Engines, Control Engineering Practice, 18(2010), 1077-1083.

Johansson, K. (1997). Relay feedback and multivariable control, Ph.D. dissertation. Dept. of Automatic Control, Lund Institute of Technology, Lund, Sweden.

Kheawhom, S. \& Bumroongsri, P. (2016). Robust gain-scheduled controller for linear parameter varying systems, ADCONP, Hiroshima.

Perez, M., \& Albertos, P. (2004). Self-oscillating and chaotic behavior of a PI-Controlled CSTR with control valve saturation, Journal of Process Control, 14, 51-59.

Seborg, D., Edgar, T., \& Mellichamp, D. (2004). Process Dynamics and Control (2 ${ }^{\text {nd }}$ Ed.). New York: John Wiley \& Sons.

Skelton, R., et al. (1997). A Unified Algebraic Approach to Linear Control Design (1 ${ }^{\text {st }}$ Ed.). Bristol: Taylor \& Francis.

Spiraxsarco Control Manuals, Spiraxsarco. (2016). Retrieved from http://www.spiraxarco.com/applications

Young, C., \& Rasmussen, B. (2008). Stable Controller Interpolation for LPV Systems, American Control Conference, Seattle, Washington, USA. 


\section{Appendix A}

\section{Proof of Theorem}

Applying Lemma (1) on the closed loop system,

$$
\left[\begin{array}{ccc}
(A+B G M) Q E_{c l}^{T}+E_{c l} Q(A+B G M)^{T} & D+B G E & E_{c l} Q(C+H G M)^{T} \\
(D+B G E)^{T} & -\gamma & (F+H G E)^{T} \\
(C+H G M) Q E_{c l}^{T} & F+H G E & -\gamma
\end{array}\right]<0
$$

where $\mathrm{G}$ is the unknown controller.

Applying the Lemma (2) on the previous LMI, it can be easily devised

$$
\begin{aligned}
& \Theta=\left[\begin{array}{ccc}
A Q E_{c l}^{T}+E_{c l} Q A^{T} & D & E_{c l} Q C^{T} \\
D^{T} & -\gamma & F^{T} \\
C Q E_{c l}^{T} & F & -\gamma
\end{array}\right] \\
& \Gamma=\left[\begin{array}{c}
B \\
0 \\
H
\end{array}\right] \quad, \Lambda=\left[M Q E_{c l}^{T} \quad E \quad 0\right]
\end{aligned}
$$

For the first condition of Lemma (2);

$$
\begin{aligned}
& \Gamma^{\perp} \Theta \Gamma^{\perp T}<0
\end{aligned}
$$

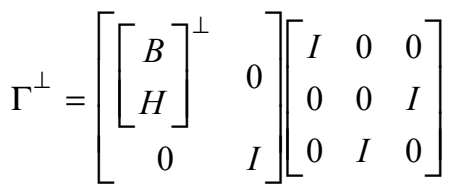

$$
\begin{aligned}
& \text { Where } \alpha=\left[\begin{array}{l}
B \\
H
\end{array}\right]^{\perp} \\
& {\left[\begin{array}{ll}
\alpha & 0 \\
0 & I
\end{array}\right]\left[\begin{array}{ccc}
I & 0 & 0 \\
0 & 0 & I \\
0 & I & 0
\end{array}\right]\left[\begin{array}{ccc}
A Q E_{c l}^{T}+E_{c l} Q A^{T} & D & E_{c l} Q C^{T} \\
D^{T} & -\gamma & F^{T} \\
C Q E_{c l}^{T} & F & -\gamma
\end{array}\right]\left[\begin{array}{ccc}
I & 0 & 0 \\
0 & 0 & I \\
0 & I & 0
\end{array}\right]\left[\begin{array}{cc}
\alpha^{T} & 0 \\
0 & I
\end{array}\right]<0} \\
& {\left[\begin{array}{cc}
\alpha & 0 \\
0 & I
\end{array}\right]\left[\begin{array}{ccc}
A Q E_{c l}^{T}+E_{c l} Q A^{T} & E_{c l} Q C^{T} & D \\
C Q E_{c l}^{T} & -\gamma I & F \\
D^{T} & F^{T} & -\gamma I
\end{array}\right]\left[\begin{array}{cc}
\alpha^{T} & 0 \\
0 & I
\end{array}\right]<0}
\end{aligned}
$$

To be compared with:

$$
\left[\begin{array}{cc}
\alpha & 0 \\
0 & I
\end{array}\right]\left[\begin{array}{cc}
\psi & \delta \\
\delta^{T} & -\gamma
\end{array}\right]\left[\begin{array}{cc}
\alpha^{T} & 0 \\
0 & I
\end{array}\right]<0
$$

After some multiplications and applying Schur's complement:

$$
\alpha \psi \alpha^{T}+\frac{1}{\gamma} \alpha \delta \delta^{T} \alpha^{T}<0
$$

Defining: 
$\alpha=\left[\begin{array}{c}B \\ H\end{array}\right]^{\perp}=\left[\left[\begin{array}{l}B_{p} \\ B_{y}\end{array}\right]^{\perp} 0\right]\left[\begin{array}{lll}I & 0 & 0 \\ 0 & 0 & I \\ 0 & I & 0\end{array}\right]$

$\psi=\left[\begin{array}{cc}A Q E_{c l}^{T}+E_{c l} Q A^{T} & E_{c l} Q C^{T} \\ C Q E_{c l}^{T} & -भ\end{array}\right]$

and Substitute to get

$\psi=\left[\begin{array}{ccc}A Y E_{p}^{T}+E_{p} Y A_{p}^{T} & A_{p} Y_{12} & E_{p} Y C_{p}^{T} \\ Y_{12}^{T} A_{p}^{T} & 0 & Y_{12}^{T} C_{p}^{T} \\ C_{p} Y E_{p}^{T} & C_{p} Y_{12} & -भ\end{array}\right]$

$Q=\left[\begin{array}{cc}Y & Y_{12} \\ Y_{12}^{T} & Y_{22}\end{array}\right]$

$\delta=\left[\begin{array}{l}D \\ F\end{array}\right]$

$\delta=\left[\begin{array}{c}D_{p} \\ 0 \\ D_{y}\end{array}\right]$

Now the first part of the first condition of lemma (2),

$$
\begin{aligned}
& \left.\alpha \Theta \alpha^{T}=\left[\left[\begin{array}{c}
B_{p} \\
B_{y}
\end{array}\right]^{\perp} 0\right]\left[\begin{array}{ccc}
I & 0 & 0 \\
0 & 0 & I \\
0 & I & 0
\end{array}\right]\left[\begin{array}{ccc}
A_{p} Y E_{p}^{T}+E_{p} Y A_{p}^{T} & A_{p} Y_{12} & E_{p} Y C_{p}^{T} \\
Y_{12}^{T} A_{p}^{T} & 0 & Y_{12}^{T} C_{p}^{T} \\
C_{p} Y E_{p}^{T} & C_{p} Y_{12} & -\gamma I
\end{array}\right]\left[\begin{array}{ccc}
I & 0 & 0 \\
0 & 0 & I \\
0 & I & 0
\end{array}\right]\left[\begin{array}{c}
B_{p} \\
B_{y}
\end{array}\right]^{\perp T}\right] \\
& \left.\left.\alpha \Theta \alpha^{T}=\left[\begin{array}{c}
B_{p} \\
B_{y}
\end{array}\right]^{\perp} 0\right]\left[\begin{array}{ccc}
A_{p} Y E_{p}^{T}+E_{p} Y A_{p}^{T} & E_{p} Y C_{p}^{T} & A_{p} Y_{12} \\
C_{p} Y E_{p}^{T} & -\gamma & C_{p} Y_{12} \\
Y_{12}^{T} A_{p}^{T} & Y_{12}^{T} C_{p}^{T} & 0
\end{array}\right]\left[\begin{array}{c}
B_{p} \\
B_{y}
\end{array}\right]^{\perp T}\right] \\
& \alpha \Theta \alpha^{T}=\left[\begin{array}{c}
B_{p} \\
B_{y}
\end{array}\right]^{\perp}\left[\begin{array}{cc}
A_{p} Y E_{p}^{T}+E_{p} Y A_{p}^{T} & E_{p} Y C_{p}^{T} \\
C_{p} Y E_{p}^{T} & -\vartheta
\end{array}\right]\left[\begin{array}{c}
B_{p} \\
B_{y}
\end{array}\right]^{\perp T}
\end{aligned}
$$

For the second part of the second condition, one gets

$$
\begin{aligned}
& \frac{1}{\gamma} \alpha \delta \delta^{T} \alpha^{T}=\frac{1}{\gamma}\left[\left[\begin{array}{c}
B_{p} \\
B_{y}
\end{array}\right]^{\perp} 0\right]\left[\begin{array}{ccc}
I & 0 & 0 \\
0 & 0 & I \\
0 & I & 0
\end{array}\right]\left[\begin{array}{c}
D_{p} \\
0 \\
D_{y}
\end{array}\right]\left[\begin{array}{lll}
D_{p}^{T} & 0 & D_{y}^{T}
\end{array}\right]\left[\begin{array}{ccc}
I & 0 & 0 \\
0 & 0 & I \\
0 & I & 0
\end{array}\right]\left[\left[\begin{array}{c}
B_{p} \\
B_{y} \\
0
\end{array}\right]^{\perp T}\right] \\
& \frac{1}{\gamma} \alpha \delta \delta^{T} \alpha^{T}=\frac{1}{\gamma}\left[\left[\begin{array}{c}
B_{p} \\
B_{y}
\end{array}\right]^{\perp} 0\right]\left[\begin{array}{ccc}
I & 0 & 0 \\
0 & 0 & I \\
0 & I & 0
\end{array}\right]\left[\begin{array}{ccc}
D_{p} D_{p}^{T} & 0 & D_{p} D_{y}^{T} \\
0 & 0 & 0 \\
D_{y} D_{p}^{T} & 0 & D_{y} D_{y}^{T}
\end{array}\right]\left[\begin{array}{ccc}
I & 0 & 0 \\
0 & 0 & I \\
0 & I & 0
\end{array}\right]\left[\left[\begin{array}{c}
B_{p} \\
B_{y}
\end{array}\right]^{\perp T}\right] \\
& \left.\frac{1}{\gamma} \alpha \delta \delta^{T} \alpha^{T}=\frac{1}{\gamma}\left[\left[\begin{array}{c}
B_{p} \\
B_{y}
\end{array}\right]^{\perp} 0\right]\left[\begin{array}{ccc}
D_{p} D_{p}^{T} & D_{p} D_{y}^{T} & 0 \\
D_{y} D_{p}^{T} & D_{y} D_{y}^{T} & 0 \\
0 & 0 & 0
\end{array}\right]\left[\begin{array}{c}
B_{p} \\
B_{y}
\end{array}\right]^{\perp T}\right] \\
& \frac{1}{\gamma} \alpha \delta \delta^{T} \alpha^{T}=\frac{1}{\gamma}\left[\begin{array}{c}
B_{p} \\
B_{y}
\end{array}\right]^{\perp}\left[\begin{array}{cc}
D_{p} D_{p}^{T} & D_{p} D_{y}^{T} \\
D_{y} D_{p}^{T} & D_{y} D_{y}^{T}
\end{array}\right]\left[\begin{array}{c}
B_{p} \\
B_{y}
\end{array}\right]^{\perp T}
\end{aligned}
$$

The $1^{\text {st }}$ condition will be as follows;

$$
\alpha \psi \alpha^{T}+\frac{1}{\gamma} \alpha \delta \delta^{T} \alpha^{T}<0
$$




$$
\left[\begin{array}{c}
B_{p} \\
B_{y}
\end{array}\right]^{\perp}\left[\begin{array}{cc}
A_{p} Y E_{p}^{T}+E_{p} Y A_{p}^{T}+\frac{D_{p} D_{p}^{T}}{\gamma} & E_{p} Y C_{p}^{T}+\frac{D_{p} D_{y}^{T}}{\gamma} \\
C_{p} Y E_{p}^{T}+\frac{D_{y} D_{p}^{T}}{\gamma} & \frac{D_{y} D_{y}^{T}}{\gamma}-\gamma
\end{array}\right]\left[\begin{array}{c}
B_{p} \\
B_{y}
\end{array}\right]^{\perp T}<0
$$

Multiplying by $\gamma$ and defining $\bar{Y}=\gamma Y$, the first condition will be;

$$
\left[\begin{array}{c}
B_{p} \\
B_{y}
\end{array}\right]^{\perp}\left[\begin{array}{cc}
A_{p} \bar{Y} E_{p}^{T}+E_{p} \bar{Y} A_{p}^{T}+D_{p} D_{p}^{T} & E_{p} \bar{Y} C_{p}^{T}+D_{p} D_{y}^{T} \\
C_{p} \bar{Y} E_{p}^{T}+D_{y} D_{p}^{T} & D_{y} D_{y}^{T}-\gamma^{2} I
\end{array}\right]\left[\begin{array}{c}
B_{p} \\
B_{y}
\end{array}\right]^{\perp T}<0
$$

For the $2^{\text {nd }}$ condition:

$$
\Lambda^{T \perp} \Theta \Lambda^{T \perp T}<0
$$

Define $N=E_{c l} Q$,

$$
\begin{aligned}
& \Lambda^{T}=\left[\begin{array}{c}
N M^{T} \\
E^{T} \\
0
\end{array}\right]
\end{aligned}
$$

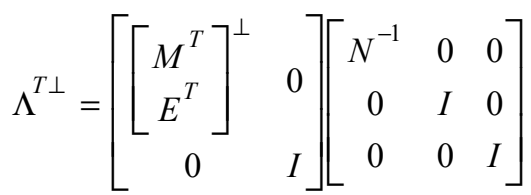

$$
\begin{aligned}
& \alpha=\left[\begin{array}{c}
M^{T} \\
E^{T}
\end{array}\right]^{\perp}=\left[\left[\begin{array}{c}
M_{p}^{T} \\
D_{z}^{T}
\end{array}\right]^{\perp} 0\right]\left[\begin{array}{lll}
I & 0 & 0 \\
0 & 0 & I \\
0 & I & 0
\end{array}\right] \\
& {\left[\begin{array}{ll}
\alpha & 0 \\
0 & I
\end{array}\right]\left[\begin{array}{ccc}
N^{-1} & 0 & 0 \\
0 & I & 0 \\
0 & 0 & I
\end{array}\right]\left[\begin{array}{ccc}
A N^{T}+N A^{T} & D & N C^{T} \\
D^{T} & -\gamma & F^{T} \\
C N^{T} & F & -\gamma
\end{array}\right]\left[\begin{array}{ccc}
N^{-T} & 0 & 0 \\
0 & I & 0 \\
0 & 0 & I
\end{array}\right]\left[\begin{array}{cc}
\alpha^{T} & 0 \\
0 & I
\end{array}\right]<0} \\
& {\left[\begin{array}{cc}
\alpha & 0 \\
0 & I
\end{array}\right]\left[\begin{array}{ccc}
N^{-1} A+A^{T} N^{-T} & N^{-1} D & C^{T} \\
D^{T} N^{-T} & -\gamma & F^{T} \\
C & F & -\gamma
\end{array}\right]\left[\begin{array}{cc}
\alpha^{T} & 0 \\
0 & I
\end{array}\right]<0}
\end{aligned}
$$

Comparing the above with the following inequality:

$$
\left[\begin{array}{cc}
\alpha & 0 \\
0 & I
\end{array}\right]\left[\begin{array}{cc}
\psi & \delta \\
\delta^{T} & -\gamma
\end{array}\right]\left[\begin{array}{cc}
\alpha^{T} & 0 \\
0 & I
\end{array}\right]<0
$$

and after some multiplications and applying schur's complement one gets:

$$
\alpha \psi \alpha^{T}+\frac{1}{\gamma} \alpha \delta \delta^{T} \alpha^{T}<0
$$

Where: 


$$
\begin{aligned}
& \psi=\left[\begin{array}{cc}
N^{-1} A+A^{T} N^{-T} & N^{-1} D \\
D^{T} N^{-T} & -\mathcal{H}
\end{array}\right] \\
& \delta=\left[\begin{array}{c}
C^{T} \\
F^{T}
\end{array}\right]
\end{aligned}
$$

Now, let's define:

$$
Q^{-1}=\left[\begin{array}{cc}
Z & Z_{12} \\
Z_{12}^{T} & Z_{22}
\end{array}\right]
$$

Now to solve the $2^{\text {nd }}$ inequality, we substitute in $\psi$ and $\delta$.

$$
\begin{aligned}
\psi & =\left[\begin{array}{ccc}
Z E_{p}^{-1} A_{p}+A_{p}^{T} E_{p}^{-T} Z & A_{p}^{T} E_{p}^{-T} Z_{12} & Z E_{p}^{-1} D_{p} \\
Z_{12}^{T} E_{p}^{-1} A_{p} & 0 & Z_{12}^{T} E_{p}^{-1} D_{p} \\
D_{p}^{T} E_{p}^{-T} Z & D_{p}^{T} E_{p}^{-T} Z_{12} & -\gamma
\end{array}\right] \\
\delta & =\left[\begin{array}{c}
C_{p}^{T} \\
0 \\
D_{y}^{T}
\end{array}\right]
\end{aligned}
$$

Hence, the first part in the $2^{\text {nd }}$ condition:

$$
\begin{aligned}
& \alpha \psi \alpha^{T}=\left[\left[\begin{array}{c}
M_{p}^{T} \\
D_{y}^{T}
\end{array}\right]^{\perp} 0\right]\left[\begin{array}{ccc}
I & 0 & 0 \\
0 & 0 & I \\
0 & I & 0
\end{array}\right]\left[\begin{array}{ccc}
Z E_{p}^{-1} A_{p}+A_{p}^{T} E_{p}^{-T} Z & A_{p}^{T} E_{p}^{-T} Z_{12} & Z E_{p}^{-1} D_{p} \\
Z_{12}^{T} E_{p}^{-1} A_{p} & 0 & Z_{12}^{T} E_{p}^{-1} D_{p} \\
D_{p}^{T} E_{p}^{-T} Z & D_{p}^{T} E_{p}^{-T} Z_{12} & -भ I
\end{array}\right]\left[\begin{array}{ccc}
I & 0 & 0 \\
0 & 0 & I \\
0 & I & 0
\end{array}\right]\left[\begin{array}{c}
M_{p}^{T} \\
D_{y}^{T} \\
0
\end{array}\right] \\
& \alpha \psi \alpha^{T}=\left[\left[\begin{array}{c}
M_{p}^{T} \\
D_{y}^{T}
\end{array}\right]^{\perp} \quad 0\right]\left[\begin{array}{ccc}
Z E_{p}^{-1} A_{p}+A_{p}^{T} E_{p}^{-T} Z & Z E_{p}^{-1} D_{p} & A_{p}^{T} E_{p}^{-T} Z_{12} \\
D_{p}^{T} E_{p}^{-T} Z & -\gamma & D_{p}^{T} E_{p}^{-T} Z_{12} \\
Z_{12}^{T} E_{p}^{-1} A_{p} & Z_{12}^{T} E_{p}^{-1} D_{p} & 0
\end{array}\right]\left[\begin{array}{c}
M_{p}^{T} \\
D_{y}^{T} \\
0
\end{array}\right] \\
& \alpha \psi \alpha^{T}=\left[\begin{array}{c}
M_{p}^{T} \\
D_{y}^{T}
\end{array}\right]^{\perp}\left[\begin{array}{cc}
Z E_{p}^{-1} A_{p}+A_{p}^{T} E_{p}^{-T} Z & Z E_{p}^{-1} D_{p} \\
D_{p}^{T} E_{p}^{-T} Z & -\gamma
\end{array}\right]\left[\begin{array}{c}
M_{p}^{T} \\
D_{y}^{T}
\end{array}\right]^{\perp T}
\end{aligned}
$$

For the $2^{\text {nd }}$ part of the $2^{\text {nd }}$ condition:

$$
\begin{aligned}
& \delta \delta^{T}=\left[\begin{array}{c}
C_{p}^{T} \\
0 \\
D_{y}^{T}
\end{array}\right]\left[\begin{array}{lll}
C_{p} & 0 & D_{y}
\end{array}\right]=\left[\begin{array}{ccc}
C_{p}^{T} C_{p} & 0 & C_{p}^{T} \\
0 & 0 & 0 \\
D_{y}^{T} C_{p} & 0 & D_{y}^{T} D_{y}
\end{array}\right] \\
& \alpha \delta \delta^{T} \alpha^{T}=\left[\left[\begin{array}{c}
M_{p}^{T} \\
D_{y}^{T}
\end{array}\right]^{\perp} 0\right]\left[\begin{array}{ccc}
I & 0 & 0 \\
0 & 0 & I \\
0 & I & 0
\end{array}\right]\left[\begin{array}{ccc}
C_{p}^{T} C_{p} & 0 & C_{p}^{T} D_{y} \\
0 & 0 & 0 \\
D_{y}^{T} C_{p} & 0 & D_{y}^{T} D_{y}
\end{array}\right]\left[\begin{array}{ccc}
I & 0 & 0 \\
0 & 0 & I \\
0 & I & 0
\end{array}\right]\left[\left[\begin{array}{c}
M_{p}^{T} \\
D_{y}^{T} \\
0
\end{array}\right]^{\perp T}\right] \\
& \left.\alpha \delta \delta^{T} \alpha^{T}=\left[\left[\begin{array}{c}
M_{p}^{T} \\
D_{y}^{T}
\end{array}\right]^{\perp} 0\right]\left[\begin{array}{ccc}
C_{p}^{T} C_{p} & C_{p}^{T} D_{y} & 0 \\
D_{y}^{T} C_{p} & D_{y}^{T} D_{y} & 0 \\
0 & 0 & 0
\end{array}\right]\left[\begin{array}{c}
M_{p}^{T} \\
D_{y}^{T} \\
0
\end{array}\right]^{\perp T}\right] \\
& \frac{1}{\gamma} \alpha \delta \delta^{T} \alpha^{T}=\frac{1}{\gamma}\left[\begin{array}{c}
M_{p}^{T} \\
D_{y}^{T}
\end{array}\right]^{\perp}\left[\begin{array}{cc}
C_{p}^{T} C_{p} & C_{p}^{T} D_{y} \\
D_{y}^{T} C_{p} & D_{y}^{T} D_{y}
\end{array}\right]\left[\begin{array}{c}
M_{p}^{T} \\
D_{y}^{T}
\end{array}\right]^{\perp T}
\end{aligned}
$$

The $2^{\text {nd }}$ condition will be: 


$$
\begin{gathered}
\alpha \psi \alpha^{T}+\frac{1}{\gamma} \alpha \delta \delta^{T} \alpha^{T}<0 \\
{\left[\begin{array}{c}
M_{p}^{T} \\
D_{z}^{T}
\end{array}\right]^{\perp}\left[\begin{array}{cc}
Z E_{p}^{-1} A_{p}+A_{p}^{T} E_{p}^{-T} Z+\frac{C_{p}^{T} C_{p}}{\gamma} & Z E_{p}^{-1} D_{p}+\frac{C_{p}^{T} D_{y}}{\gamma} \\
D_{p}^{T} E_{p}^{-T} Z+\frac{D_{y}^{T} C_{p}}{\gamma} & \frac{D_{y}^{T} D_{y}}{\gamma}-\gamma
\end{array}\right]\left[\begin{array}{c}
M_{p}^{T} \\
D_{z}^{T}
\end{array}\right]^{\perp T}<0}
\end{gathered}
$$

Multiplying by $\gamma$ and defining $X=\gamma Z$, the $2^{\text {nd }}$ condition will be;

$$
\left[\begin{array}{c}
M_{p}^{T} \\
D_{z}^{T}
\end{array}\right]^{\perp}\left[\begin{array}{cc}
X E_{p}^{-1} A_{p}+A_{p}^{T} E_{p}^{-T} X+C_{p}^{T} C_{p} & X E_{p}^{-1} D_{p}+C_{p}^{T} D_{y} \\
D_{p}^{T} E_{p}^{-T} X+D_{y}^{T} C_{p} & D_{y}^{T} D_{y}-\gamma^{2} I
\end{array}\right]\left[\begin{array}{c}
M_{p}^{T} \\
D_{z}^{T}
\end{array}\right]^{\perp T}<0
$$

For the $3^{\text {rd }}$ condition:

$$
\begin{aligned}
Q^{-1} & =\left[\begin{array}{cc}
Z & Z_{12} \\
Z_{12}^{T} & Z_{22}
\end{array} \mid=P>0\right. \\
Q & =\left[\begin{array}{cc}
Y & Y_{12} \\
Y_{12}^{T} & Y_{22}
\end{array}\right]
\end{aligned}
$$

So one can conclude that;

$$
\begin{aligned}
& Y=\left(Z-Z_{12} Z_{22}^{-1} Z_{12}^{T}\right)^{-1} \\
& Z-Y^{-1}=Z_{12} Z_{22}^{-1} Z_{12}^{T}>0 \\
& {\left[\begin{array}{ll}
Z & I \\
I & Y
\end{array}\right]>0}
\end{aligned}
$$

Finally, multiply by $\gamma$ to get:

$$
\begin{aligned}
& {\left[\begin{array}{ll}
\gamma Z & \gamma \\
\gamma & \gamma Y
\end{array}\right]>0} \\
& {\left[\begin{array}{ll}
X & \gamma \\
\gamma & \bar{Y}
\end{array}\right]>0}
\end{aligned}
$$

\section{Copyrights}

Copyright for this article is retained by the author(s), with first publication rights granted to the journal.

This is an open-access article distributed under the terms and conditions of the Creative Commons Attribution license (http://creativecommons.org/licenses/by/4.0/). 